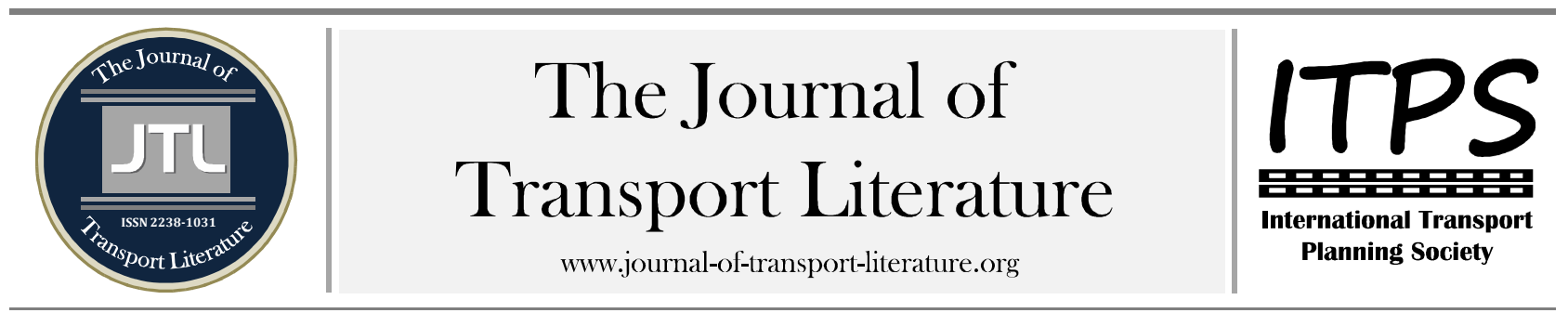

\title{
Programação de caminhões de múltiplos tipos no transporte de derivados de petróleo para a construção de rodovias
}

José Luciano Lopes da Costa Filho+; Bruno de Athayde Prata

Federal University of Ceará, Fortaleza, Brazil

\section{Article Info}

Keywords:

programação de caminhões dimensionamento de frota programação inteira word4

Submitted 30 Nov 2014;

received in revised form 13 Jan 2015 . accepted 18 Jan 2015

Licensed under

Creative Common

CC-BY $3.0 \mathrm{BR}$

\section{Abstract}

0 problema de programação de caminhões é um tema de grande relevância na gestão de frota das empresas. Estas enfrentam dificuldades em gerenciar seus veículos devido às diversas variáveis inerentes ao processo, tais como o tamanho ideal da frota, os diversos tipos de caminhões disponíveis, a capacidade de carga do caminhão, as informações técnicas do cliente e o agendamento das viagens. No que se refere ao transporte de derivados de petróleo, existem diversas características operacionais que dificultam a programação de caminhões. 0 presente trabalho tem como objetivo desenvolver um modelo de programação inteira para a otimização da programação de veículos de múltiplos tipos para o transporte de derivados de petróleo para obras de construção de rodovias. Foi desenvolvido um modelo que buscasse a minimização da frota de caminhões disponíveis. Como conclusões, pode-se ressaltar que a metodologia empregada serviu para minimizar em $23,3 \%$, em media, a frota necessária no período analisado. 0 desenvolvimento de indicadores de desempenho permitiu avaliar a qualidade das soluções geradas.

+ Corresponding author. Universidade Federal do Ceará, Centro de Tecnologia. Campus do Picí, Bloco 710, Pici. 60455760 - Fortaleza, CE - Brasil.

E-mail address: lucianocostta@yahoo.com.br.

\section{Introdução}

O estudo da programação de veículos para o transporte de asfalto e derivados é de grande importância para as empresas do setor, pois uma alocação inadequada pode acarretar em alguns problemas, tais como acréscimo nos custos operacionais, aumento dos tempos de entrega dos produtos e atraso no retorno dos veículos, bem como em maior ociosidade da frota. 0 atraso mencionado é referente ao erro na alocação de um caminhão com destino a uma determinada obra, cuja demanda é menor que a carga transportada. Assim, é provável que o veículo fique algum tempo na obra, até descarregar todo o produto, ocasionando atraso em seu retorno. 0 desenvolvimento de métodos para determinação da frota ótima contribuirão para redução da ociosidade da frota, bem como para evitar atrasos no retorno dos veículos para a origem.

O desenvolvimento da programação de caminhões para as diversas obras de construção de rodovias e o estudo de suas particularidades é fundamental para a melhoria da gestão de uma transportadora deste segmento. As consequências seriam expressas em termos da redução de custos operacionais, da redução dos tempos de viagem entre refinaria e obra ou entre usina e obra, bem como da minimização das incompatibilidades existentes entre as viagens, tais como o problema relacionado ao tamanho dos tanques (tanto dos caminhões quanto das obras).

Para a resolução da programação de veículos em sistemas de transporte público, diversas abordagens têm sido reportadas na literatura. Métodos exatos e heurísticas são apresentadas por Carraresi e Gallo (1984), Beaujon e Turnquist (1991), Daduna e Paixão (1995), Freling e Paixão (1995), Freling et al. (1999), Baita et al. (2000), Haghani et al. (2003), Wang e Shen (2007) e Eliiyi et al. (2008). Para o caso em que são permitidos veículos de múltiplos tipos, podem ser citados Ferland e Michelon (1988) e Ceder (2011). Para a modelagem da programação de caminhões no transporte de cargas, podem ser citados os trabalhos de Powell et al. (1984), Bausch et al. (1994), Hatori (2000), Morabito e Vasco (2011), Stojanovic et al. (2011). No transporte de concreto usinado para obras de construção civil, algumas abordagens são apresentadas por Feng et al. (2004), Feng e Wu (2006), Yan e Lai (2007), Lin et al. (2010), Choi e Nieto (2011), Yan et al. (2011) e Cortes e Cunha (2014).

Embora existam diversos trabalhos abordando o tema da programação de veículos em sistemas de transporte público e no transporte de cargas, ainda são escassos os trabalhos que abordem o tema da programação de veículos no transporte de derivados de petróleo para a construção de rodovias. Ademais, modelos que considerem as peculiaridades operacionais de caminhões-tanque não foram encontrados na literatura.

O objetivo do trabalho reportado neste artigo é desenvolver um modelo de Programação Inteira para a otimização da programação de veículos para o transporte de derivados de petróleo para obras de construção de rodovias, buscando a redução da frota disponível. Ressalta-se que a abordagem proposta proporciona benefícios para o sistema de transporte de derivados de petróleo, que vão além da redução dos custos, passando pelo planejamento adequado dos veículos para carregamento conforme dimensões da carreta e a demanda do cliente, evitando a designação de um veículo cuja capacidade 
não é adequada para o atendimento do pedido. É necessário evitar que a carreta alocada para o atendimento de um pedido tenha capacidade inferior ao pedido do cliente.

0 trabalho é composto por mais três seções, descritas a seguir. Na próxima, é apresentada uma nova formulação proposta para a programação de caminhões de múltiplos tipos. Na terceira seção é apresentado o estudo de caso, contendo os resultados computacionais obtidos, em instâncias adaptadas na literatura e em uma empresa real do setor de transporte de cargas. Por fim, apresentam-se algumas conclusões, e possíveis desenvolvimentos futuros para o trabalho.

\section{0 problema de programação de veículos com múltiplos tipos}

0 problema de programação de veículos consiste em um conjunto de viagens $T$ que devem ser executadas por um determinado número de veículos n. Cada viagem i $(1 \leq i \leq n)$ tem características de tempo e espaço (localização) específicas, tais como: (i) um ponto de partida $S L_{i}$, (ii) um ponto de chegada $E L_{i}$, (iii) um tempo de início $S T_{i}$.

Em alguns casos, o tempo de início não é fixo, mas pode pertencer a um intervalo de tempo dado [ai, bi], que é denominado janela de tempo. Sendo assim, para cada tarefa $i, a_{i}$ e $b_{i}$ são o tempo de início mais cedo e o tempo de início mais tarde, respectivamente. Seja $q_{i}$ a demanda do cliente i, ressalta-se, também, que o serviço i só poderá ser executado por um veículo com capacidade de carga acima de $q_{i}$. Cada veículo de tipo $k(1 \leq k \leq K)$ é caracterizado pela sua capacidade de carga $h_{k}$.

Para executar a viagem $i$, um veículo de tipo $k$ precisa estar em um ponto $S L_{i}$ no tempo $S T_{i}$, e tal veículo necessita de $D_{i k}$ unidades de tempo para executar o serviço demandado. Sendo assim, no tempo $S T_{i}+D_{i k}$ o veículo estará em $E L_{i}$, que deve ser o destino da viagem, e disponível para executar outras tarefas. Na sequência, o veículo pode executar o serviço $j$ se, levando-se em consideração o tempo $t_{i j k}$ necessário para o veículo sair de $E L_{i}$ para $S L_{j}$, o mesmo estiver disponível no tempo $S T_{j}$ no ponto de partida $S L_{j}$, ou seja,

$$
S T_{i}+D_{i k}+t_{i j k} \leq S T_{j}
$$

Vale destacar ainda que as viagens estão listadas em uma tabela de horários, em que: (i) cada viagem é realizada por um veículo, (ii) um dado número de restrições é satisfeito, (iii) uma função custo é minimizada.

Assume-se, portanto, que é dada uma tabela de horários, contendo um conjunto de viagens $\mathrm{V}=\left\{v_{1}, v_{2}, \ldots, v_{n}\right\}$, onde cada viagem vi é definida como segue:

- $\zeta_{i}$, o tempo de início da viagem $i$;

- $l_{i}$, a duração da viagem $i$;

- $\tau_{\mathrm{ij}}$, duração da viagem não-produtiva (deadhead) entre os pontos $i$ e $j$;

- $o_{i}$, a origem ou ponto de partida da viagem $i$;

- $d_{i}$, o destino ou ponto de chegada da viagem $i$.

0 par $\left(v_{i}, v_{j}\right)$ vai ser compatível se:

$$
\zeta_{i}+l_{i}+\tau_{i j}+\varepsilon \leq \zeta_{i}
$$

em que $\varepsilon \geq 0$ é um parâmetro para considerar possíveis atrasos na realização de uma dada viagem.

$\mathrm{Na}$ revisão de literatura realizada, não foram encontrados trabalhos que utilizassem modelos matemáticos para programação de veículos para o suprimento de obras de pavimentação.

Neste trabalho, busca-se suprir esta escassez, considerando algumas características práticas do problema, preservando a utilidade do modelo.

É importante apresentar algumas notações para o modelo proposto, conforme segue:

Conjuntos

- $N$ - clientes

- $K$ - veículos

Parâmetros

- $\mathrm{c}_{i j k}$ - custo de transporte no arco (i, j) pelo caminhão de tipo $\mathrm{k}$.

Para valores de $c_{i j k}$ iguais a 0 ou 1 , o objetivo é reduzir o custo capital. Assim, tem-se o equivalente a minimizar o tamanho da frota. Para valores diferentes de 0 e 1 o objetivo é reduzir os custos operacionais.

Neste problema, busca-se minimizar a frota de caminhões destinada a atender um conjunto de clientes. Devem ser atendidos todos os clientes utilizando os recursos (caminhões) disponíveis.

Um modelo matemático para este problema pode ser elaborado utilizando um grafo $\left(N_{k}, A_{k}\right)$ das viagens associadas com os veículos de tipo $k$, sendo caracterizado da seguinte forma:

com cada viagem $i$, na qual $q_{i} \leq h_{k}$, associa à um nó $i \in N_{k}$. Dois nós, s e t, são associados à usina para indicar onde os veículos iniciam e finalizam a viagem;

um arco (i, j) existe, isto é, (i, j) $\in \mathrm{A}_{k}$, ou o serviço $j$ pode ser executado após o serviço $i$ por um veículo de tipo $k$, se

$$
a_{i}+D_{i k}+\mathrm{t}_{i j k} \leq \mathrm{b}_{j}
$$

Além disso, para cada serviço $i \in N_{k}$, devem ser introduzidos os $\operatorname{arcos}(\mathrm{s}, \mathrm{i})$ e (i, t) em $\mathrm{A}_{k}$, permitido que $i$ seja a primeira ou a última tarefa em uma sequência, respectivamente. $\left.A_{k}\right\}$.

Seja $\Lambda_{i}=\left\{k: q_{i} \leq h_{k}, 1 \leq k \leq K\right\}, \overline{\mathrm{A}}_{k}=\left\{(\mathrm{i}, \mathrm{j}) \in A_{k}: i \neq \mathrm{s}, \mathrm{t} ; j \neq \mathrm{s}, \mathrm{t}\right\}$. Para $i \in N_{k}, P_{i k}=\left\{J \in N_{k}:(\mathrm{i}, \mathrm{j}) \in A_{k}\right\}$ e $S_{i k}=\left\{J \in N_{k}:(\mathrm{i}, \mathrm{j}) \in\right.$

Para este grafo, tem-se que os nós estão agrupados em caminhos ou sequências, com cada uma iniciando em s e terminando em t. As restrições da janela de tempo devem ser satisfeitas para todas as tarefas e o objetivo é minimizar o custo de capital. 0 modelo matemático pode ser enunciado como segue:

Sujeito a:

$$
\text { Minimizar z }=\sum_{k=1}^{k} \sum_{(i, j) \in A_{k}} c_{i j k} x_{i j k}
$$

$$
\begin{array}{ll}
\sum_{k \in \Lambda_{i}} y_{i k}=1 & 1 \leq i \leq n \\
\sum_{i \in P_{j k}} x_{i j k}=y_{j k} & j \in N_{k}-\{\mathrm{s}, \mathrm{t}\}, 1 \leq k \leq K \\
\sum_{j \in S_{i k}} x_{i j k}=y_{i k} & i \in N_{k}-\{\mathrm{s}, \mathrm{t}\}, 1 \leq k \leq K
\end{array}
$$




$$
\begin{aligned}
& \sum_{k \in K} h_{k} y_{i k}=q_{i} \quad 1 \leq i \leq n \\
& x_{i j k}>0 \\
& a_{i} \leq S T_{i} \leq b_{i} \text {, } \\
& x_{i j k} \in\{0,1\} \\
& S T_{i}+D_{i k}+t_{i j k} \leq S T_{j} \\
& ,(i, j) \in A_{k}, 1 \leq k \leq K \\
& 1 \leq i \leq n \\
& y_{i k} \in\{0,1\} \\
& \text { (i, j) } \in A_{k}, 1 \leq k \leq K
\end{aligned}
$$

A função objetivo (4) a ser minimizada representa o custo de capital dos veículos, de múltiplos tipos. 0 conjunto de restrições do tipo (5) impõe que cada cliente é atendido por um veículo de um dado tipo. Os conjuntos de restrições dos tipos (6) e (7) garantem que cada cliente é atendido por apenas um veículo. 0 conjunto de restrições do tipo (8) assegura que cada cliente será atendido por um veículo cuja capacidade é igual à sua demanda. Os conjuntos de restrições dos tipos (9) e (10) dizem respeito às janelas de tempo. Por fim, os conjuntos de restrições dos tipos (11) e (12) dizem respeito à definição das variáveis de decisão.

De acordo com Ceder (2011), o problema de programação de veículos com múltiplos tipos é pertencente à classe de problemas NP-difícil. Deste modo, não existe garantia de que métodos exatos podem obter soluções ótimas para o problema em tempo computacional admissível. No caso prático que será estudado no presente trabalho, pode-se observar que a quantidade de clientes e a quantidade de tipos de veículos são da ordem de grandeza de dezenas, de modo que a quantidade de variáveis de decisão binárias é da ordem de poucas centenas. Assim, espera-se que o uso de métodos exatos possa obter soluções ótimas em tempo computacional admissível.

\section{Estudo de Caso}

0 estudo de caso foi realizado em uma empresa real, que solicitou que seu nome não fosse identificado. A empresa em estudo é uma transportadora de derivados de petróleo que atua distribuindo produtos em obras de construção de rodovias situadas nas regiões Norte e Nordeste do Brasil. Em sua sede, há um escritório onde funcionam o setor administrativo e o almoxarifado. No mesmo local ainda existem a garagem para os caminhões e a oficina.

As únicas ferramentas de gestão logística eram as planilhas elaboradas para o controle da frota, dos motoristas e das obras, além do rastreador para todos os veículos. No entanto, estes controles eram limitados e estas ferramentas não contribuíam para um scheduling eficiente e automatizado, havendo imprecisão ao realizar a programação das viagens e a designação dos caminhões adequados para o atendimento.

Dados reais sobre os pedidos, referentes aos meses de abril a junho de 2013, foram coletados. A transportadora possui frota própria e heterogênea para o transporte de asfalto, sendo composta de 20 caminhões de variadas capacidades de carregamento, conforme descrito a seguir: 8 caminhões de 25 toneladas, 6 caminhões de 30 toneladas, 2 caminhões de 33 toneladas, 3 caminhões de 35 toneladas e 1 caminhão de 50 toneladas.

0 modelo matemático proposto foi aplicado por meio dos dados inseridos em uma tabela de horários contendo informações dos pedidos dos clientes que foram coletadas junto a uma empresa do setor durante o período de nove semanas de trabalho. As informações da tabela são referentes às demandas dos clientes, contendo o devido destino de cada carga, a quantidade de carga solicitada, os tempos médios de início e duração das viagens, além de informações dos pedidos, que deverão ser distribuídos entre os caminhões que compõem a frota da empresa estudada.

É importante ressaltar que, na prática da empresa em estudo, conforme relatado por profissionais da mesma, a programação é realizada semanalmente, ou seja, o gestor de frota recebe o relatório com os pedidos diretamente do responsável comercial da distribuidora e ambos fazem o planejamento dos carregamentos da semana seguinte, sempre analisando os caminhões disponíveis e suas respectivas capacidades de carga, bem como o tamanho do pedido colocado pelo cliente.

Os resultados apresentados na Tabela 1 foram obtidos no software CPLEX® versão 12.6, executado em um sistema operacional Windows 7 de 64 Bits, com processador Intel Pentium 1,87 GHz e 4 GB de memória RAM.

$$
\text { Tabela } 1 \text { - Resultados computacionais }
$$

\begin{tabular}{c|c|c|c|c|c}
\hline $\begin{array}{c}\text { Instância } \\
\text { (Semana) }\end{array}$ & $\begin{array}{c}\# \\
\text { Viagens }\end{array}$ & $\begin{array}{c}\# \\
\text { Caminhões } \\
\text { disponíveis }\end{array}$ & $\begin{array}{c}\text { Solução } \\
\text { ótima } \\
\text { (\#caminhões) }\end{array}$ & $\begin{array}{c}\text { Economia } \\
\text { de frota }\end{array}$ & $\mathrm{t}(\mathrm{s})$ \\
\hline S1 & 29 & 20 & 15 & $25 \%$ & 0,22 \\
\hline S2 & 31 & 20 & 15 & $25 \%$ & 0,25 \\
\hline S3 & 34 & 20 & 16 & $20 \%$ & 0,47 \\
\hline S4 & 24 & 20 & 13 & $35 \%$ & 0,19 \\
\hline S5 & 24 & 20 & 15 & $25 \%$ & 0,30 \\
\hline S6 & 49 & 20 & 16 & $20 \%$ & 0,98 \\
\hline S7 & 47 & 20 & 16 & $20 \%$ & 0,75 \\
\hline S8 & 53 & 20 & 17 & $15 \%$ & 1,03 \\
\hline S9 & 43 & 20 & 15 & $25 \%$ & 0,62 \\
\hline
\end{tabular}

A economia de frota é calculada pela diferença entre o número de veículos disponíveis e o número de veículos utilizados na semana, dividido pelo número total de veículos, sendo medida em valores percentuais.

Foi possível verificar, pela Tabela 1, que em todas as semanas analisadas houve economia da frota, com redução média de $23,3 \%$, ou seja, nem todos os caminhões disponíveis foram utilizados para a realização das viagens. Ressalta-se que, na prática, a inexistência de controles mais apurados de gestão de frota resultava na utilização de todos os veículos durante uma semana de trabalho e, em algumas vezes, a má alocação do veículo a uma determinada viagem permitia que a seguinte situação ocorresse: um caminhão disponível no pátio, um pedido de carregamento pendente e a incompatibilidade entre a capacidade deste veículo e a demanda do cliente. 
É importante mencionar que não há informações das programações semanais reais que foram executadas. Isto significa que após o planejamento das viagens, as alterações existentes e a execução das mesmas, não há dados de como as demandas foram atendidas.

Obteve-se, assim, auxílio na tomada de decisão, pois foi permitido selecionar o veículo correto para a obra correta, evitando desgastes com o cliente. 0 balanceamento das viagens, entretanto, poderia ser efetuado para equilibrar a quantidade de viagens realizadas pelos veículos de mesmo tipo, bem como a produtividade dos motoristas. A Figura 1 apresenta o percentual de utilização dos vinte caminhões da frota na execução das viagens ao longo das nove semanas de trabalho e visa demonstrar a questão do balanceamento das cargas para os caminhões da frota.

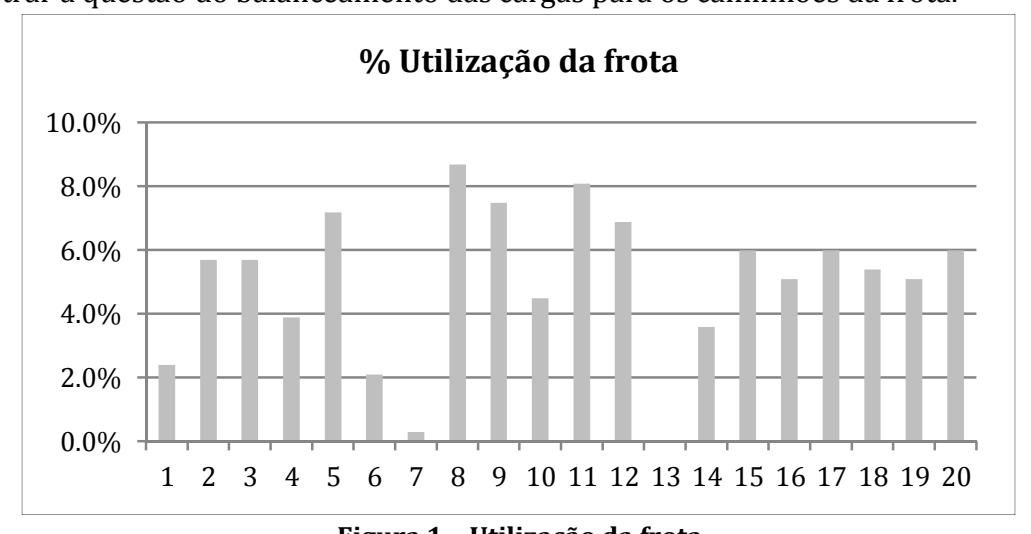

Figura 1 - Utilização da frota

Fonte: autores

Com exceção dos veículos 7 e 13, que tiveram pouca ou nenhuma utilização, os demais caminhões são utilizados de forma razoável ao longo dos períodos analisados. 0 caminhão 1 é especial por ser o único do tipo 50 toneladas e recebe poucos pedidos. Os veículos 5 e 6 são do tipo 33 toneladas e o modelo optou por utilizar em maior escala o primeiro, já que não há nenhuma regra para distribuição balanceada das viagens.

\section{Conclusão}

Este trabalho resultou na elaboração de um modelo que buscasse a minimização da frota de caminhões disponíveis no transporte de derivados de petróleo na construção de rodovias. 0 modelo de Programação Inteira foi implementado no software CPLEX® e foi possível constatar que a metodologia empregada no trabalho serviu para minimizar a frota disponível, ou seja, permitiu gerar programações de caminhões para o transporte de derivados na construção de rodovias com custo de capital mínimo. Permitiu, ainda, a automatização da geração de programações, reduzindo substancialmente os tempos de planejamento e garantindo a viabilidade das programações geradas.

Como propostas para estudos futuros, verifica-se a necessidade de trabalhar melhor o balanceamento das viagens, pois se torna importante para equilibrar a quantidade de viagens realizadas pelos veículos de mesmo tipo, bem como para calcular a produtividade dos motoristas. Outra sugestão para estudos futuros seria considerar múltiplos critérios, tais como custo, tempo, ociosidade da frota, folgas entre viagens e balanceamento das viagens entre os caminhões.

Ainda é possível considerar o produto carregado e as particularidades de cada um, tais como a possibilidade de um caminhão carregar o produto X após ter carregado o produto $\mathrm{Y}$ na viagem anterior. Ainda, avaliar a necessidade de fazer a programação semanal dos caminhões levando em consideração os tipos de produtos que cada veículo deverá carregar.

Na prática, a geração de serviços de motoristas não atende à legislação vigente, já que se torna difícil controlar os horários dos motoristas quando os mesmos estão em viagem, com dificuldade de acesso ao telefone ou mesmo de visualizar o veículo no roteirizador, já que há obras em regiões remotas. A orientação é incorporar a programação dos motoristas (crew scheduling) na programação dos caminhões.

\section{Referências}

Baita, F., Pesenti, R., Ukovich, W., \& Favaretto, D. (2000). A comparison of different solution approaches to the vehicle scheduling problem in a practical case. Computers \& Operations Research, 27(13), 1249-1269.

Bausch, D., Brown, G., \& Ronen, D. (1994). Dispatching shipments at minimal cost with multiple mode alternatives. Journal of Business Logistics, 15(1).

Beaujon, G. ,\& Turnquist, M. (1991). A model for fleet sizing and vehicle allocation. Transportation Science, 25(1), 19-45.

Carraresi, P., \& Gallo, G. (1984). Network models for vehicle and crew scheduling. European Journal of Operational Research, 16.

Ceder, A. (2011). Public-transit Vehicle Schedules Using a Minimum Crew-cost Approach. Total Logistic Management, 4, 21-42.

Choi, Y., \& Nieto, A. (2011). Optimal Haulage Routing of Off-road Dump Trucks in Construction and Mining Sites Using Google Earth and a Modified Least-cost Path Algorithm. Journal of Automation in Construction, 20(7), 982-997.

Cortes, C. S., \& Cunha, C. B. (2014). Sistema de apoio à decisão baseado em planilha eletrônica para otimização da programação de entrega de concreto pronto. Journal of Transport Literature, 8, 125-158.

Daduna, J. R., \& Paixão, J. M. P. (1995). Vehicle Scheduling for public mass transit - an Overview. In: Daduna, J. R., Branco, I., \& Paixão, J. M. P. (Eds.) Computer-Aided Transit Scheduling. Lecture Notes in Economics and Mathematical Systems, Springer, 430, 76-90.

Eliiyi, D., Ornek, A., \& Karakütük, S. (2009). A vehicle scheduling problem with fixed trips and time limitations. International Journal of Production Economics, 117(1), 150-161.

Feng, C., Cheng, T., \& Wu, H. (2004). Optimizing the schedule of dispatching RMC trucks through genetic algorithms. Automation in Construction, 13(3), 327-340.

Feng, C., \& Wu, H. (2006). Integrating fmGA and cyclone to optimize the schedule of dispatching RMC trucks. Automation in Construction.

Ferland, J. A., \& Michelon, P. (1988). The Vehicle Scheduling Problem with Multiple Vehicle Types. Journal of the Operational Research Society, 39(6), 577-583.

Freling, R., \& Paixão, J. M. P. (1995). Vehicle scheduling with time constraint. In Daduna, J., Branco, I., \& Paixão, J. M. P. (eds.) Computer-aided transit scheduling. Lecture Notes in Economics and Mathematical Systems, 430, 130-144. 
Freling, R., Wagelmans, A. P. M., \& Paixão, J. M. P. (1999). An overview of models and techniques for integrating. In: Wilson, N. H. M. (Ed.) Computer-aided Transit Scheduling, Lecture Notes in Economics and Mathematical Systems, Springer, 471, 441-460.

Haghani, A., \& Banihashemi, M. (2003). A comparative analysis of bus transit vehicle scheduling models. Transportation Research Part B, $37,301-322$

Hatori, S. Truck Dispatch Scheduling by Constraint Logic Programming (2000). System Plaza Inc., Supply Chain Division. APDSI.

Lin, P., Wang, J., Huang, S., \& Wang, Y. (2010). Dispatching Ready Mixed Concrete Trucks Under Demand Postponement And Weight Limit Regulation. Journal of Automation in Construction, 19(6), 798-807.

Morabito, R., \& Vasco, R. (2011). Modelos Para o Dimensionamento e Alocação Dinâmica de Veículos no Transporte Rodoviário de Cargas Completas Entre Terminais. XXXI Encontro Nacional de Engenharia de Produção: ENEGEP.

Stojanovic, D., Nikolicic, S., \& Milicic, M. (2011). Transport fleet sizing by using make and buy decision-making. Economic Annals, LVI(190).

Wang, H., \& Shen, J. (2007). Heuristic Approach for Solving Transit Vehicle Scheduling Problem With Route and Fueling Time Constraints. Journal of Applied Mathematics and Computation, 190(2), 1237-1249.

Yan, S., \& Lai, W. (2007). An Optimal Scheduling Model for Ready Mixed Concrete Supply With Overtime Considerations. Automation in Construction, 16(6), 734-744.

Yan, S., Lin, H., \& Liu, Y. (2011). Optimal schedule adjustments for supplying ready mixed concrete following incidents. Automation in Construction, 20(8), 1041-1050.

\section{Abstract}

The truck scheduling problem is an important topic in the companies' fleet management. Many companies face difficulties to manage their vehicles due to several variables inherent to the management process, such as the optimal fleet size, multiple types of trucks available, trucks capacity, the technical information from the client's construction site and trips scheduling. In terms of the transportation of oil products, there are many operational characteristics that make the truck scheduling difficult. The purpose of this research study was to develop an Integer Programming model to optimize the multi-type truck scheduling for the transportation of oil products for road construction sites. A model that aimed to minimize the truck fleet available was developed. The method used has minimized the available fleet in $23,3 \%$, in average, in the period under review. The development of key performance indicators allows to evaluate the quality of the solutions created.

Key words: truck scheduling, fleet size and mix, integer programming. 